\section{Polar codes for distributed source coding}

\section{S. Önay}

A polar coding method to construct a distributed source coding scheme which can achieve any point on the dominant face of the Slepian-Wolf rate region for sources with uniform marginals is proposed. Source encoding and decoding operations are performed using efficient algorithms which makes practical implementation feasible. Simulation results are given to exhibit the performance of the presented method.

Introduction: Slepian-Wolf (SW) coding [1] refers to the distributed compression of a memoryless source pair $(X, Y)$ sampled from a known distribution $\mathcal{P}_{X, Y}(x, y)$. The problem setting assumes separate encoding but joint decoding of sources $X$ and $Y$. The SW theorem states that as long as the source rate pair $\left(R_{X}, R_{Y}\right)$ is inside the achievable rate region ( $\mathrm{SW}$ rate region) error-free compression is possible. The SW rate region is described by the inequalities $R_{X} \geq H(X \mid Y)$, $R_{Y} \geq(Y \mid X)$ and $R_{X}+R_{Y} \geq H(X, Y)$. The corner points $\left(R_{X}, R_{Y}\right)=$ $(H(X \mid Y), H(Y))$ and $\left(R_{X}, R_{Y}\right)=(H(X), H(Y \mid X))$ on the achievable rate boundary are also referred to as 'asymmetric' operating points. And any point on the line segment between these corner points ('dominant face'), where $R_{X}+R_{Y}=H(X, Y)$, is also referred to as a 'nonasymmetric' operating point

Starting with the pioneering work of Pradhan and Ramchandran [2], an extensive literature on applying channel codes for practical implementation of SW coding has been developed. Schemes utilising turbo and LDPC codes in both asymmetric [3-5] and non-asymmetric $[6,7]$ SW problems were constructed. Polar coding [8], recently discovered by Arkkan, is the first provably capacity-achieving coding method with low encoding and decoding complexity for the class of binary-input discrete memoryless channels. Shortly after its discovery, a number of works have been published which showed that polar codes are also provably optimal for source coding, asymmetric Slepian-Wolf and Wyner-Ziv problems $[9,10]$.

This Letter proposes a method to achieve any point on the dominant face of the Slepian-Wolf (SW) achievable rate region using polar codes for the case of sources with uniform marginals. The method is based on the framework of [11] and a systematic version of polar codes [12]. Source decoding is performed using polar decoders which yield computationally efficient implementation. Experimental results are presented to demonstrate the effectiveness of the coding scheme. We use a successive cancellation list (SCL) decoder [13] with CRC to achieve the best possible results.

Proposed method: We follow the method of [11] to construct a nonasymmetric SW setting using polar codes. $X$ and $Y$ are assumed to be binary RVs with uniform marginals. The correlation model between sources $X$ and $Y$ is given as $Y=X \oplus E$, where $E \sim \operatorname{Bernoulli}(\varepsilon)$. Thus, $H(X \mid Y)=H(Y \mid X)=H(E)=\mathcal{H}(\varepsilon)$, where $\mathcal{H}(\varepsilon)=-\varepsilon \log \varepsilon-$ $(1-\varepsilon) \log (1-\varepsilon)$. Here, $Y$ can also be viewed as a version of $X$ passed through a virtual BSC with cross-over probability $\varepsilon$.

The method of [11] can be summarised as follows. Consider two I.I.D. distributed and correlated $N$-vectors $\mathbf{x}=\left[\begin{array}{l}\mathbf{x}^{a} \\ \mathbf{x}^{b}\end{array}\right]$ and $\mathbf{y}=\left[\begin{array}{ll}\mathbf{y}^{a} & \mathbf{y}^{b}\end{array}\right]$ sampled from source $\mathrm{RV}(X, Y)$. $\mathbf{x}^{a}$ represents the first $K$ bits and $\mathbf{x}^{b}$ represents the last $N-K$ bits of vector $\mathbf{x}$ (the same applies to $\mathbf{y}$ ). Also, let $\mathbf{x}^{a}=\left[\begin{array}{ll}\mathbf{x}_{1}^{a} & \mathbf{x}_{2}^{a}\end{array}\right]$ and $\mathbf{y}^{a}=\left[\begin{array}{ll}\mathbf{y}_{1}^{a} & \mathbf{y}_{2}^{a}\end{array}\right] . \mathbf{x}_{1}^{a}$ represents the first $K_{1}$ bits and $\mathbf{x}_{2}^{a}$ represents the last $K_{2}$ bits of $\mathbf{x}^{a}$ (the same applies to $\mathbf{y}^{a}$ ), where $K_{1}+K_{2}=K$. Let $G$ be a $K \times N$ generator matrix and $H$ a $(N-K) \times N$ parity check matrix of some block code. Assume that $H$ has the form $\left[H_{a} H_{b}\right]$ where $H_{a}$ is an $(N-K) \times K$ matrix and $H_{b}$ is an $(N-K) \times(N-K)$ non-singular matrix. Notice that the systematic version of a code is a special case with $H_{b}=I_{N-K}$. The syndromes of $\mathbf{x}$ and $\mathbf{y}$ are calculated as $\mathbf{s}_{\mathbf{x}}=\mathbf{x} H^{T}=\mathbf{x}^{a} H_{a}^{T} \oplus \mathbf{x}^{b} H_{b}^{T}$ and $\mathbf{s}_{\mathbf{y}}=\mathbf{y} H^{T}=\mathbf{y}^{a} H_{a}^{T} \oplus \mathbf{y}^{b} H_{b}^{T}$, respectively. Then, the $X$-encoder sends $\left(\mathbf{x}_{1}^{a}, \mathbf{s}_{\mathbf{x}}\right)$ and the $Y$-encoder sends $\left(\mathbf{y}_{2}^{a}, \mathbf{s}_{\mathbf{y}}\right)$. The total number of bits sent by both encoders is $2 N-K$ yielding a sum rate $R=2-K / N$. By choosing $K / N=2-H(X, Y)=1-H(E)$, this scheme results in a code operating on the dominant face of the SW region. Then by varying $K_{1}$ and $K_{2}$ subject to $K_{1}+K_{2}=K$, one can operate at any point on the dominant face.

The decoding of the above scheme, which is depicted in Fig. 1, is performed as follows. Let $\mathbf{e}=\mathbf{x} \oplus \mathbf{y}$ be the error vector. Then, $\mathbf{s}_{\mathbf{e}}=\mathbf{e} H^{T}=(\mathbf{x} \oplus \mathbf{y}) H^{T}=\mathbf{s}_{\mathbf{x}} \oplus \mathbf{s}_{\mathbf{y}}$. The method assumes that there is a syndrome decoder for the given code which is supplied with an all-zeros vector as input and $\mathbf{s}_{\mathbf{e}}$ as the coset index. The estimate $\hat{\mathbf{e}}$ is obtained as the output. With this estimated error pattern, $\mathbf{x}_{2}^{a}$ and $\mathbf{y}_{1}^{a}$ can be recovered using $\mathbf{y}_{2}^{a}$ and $\mathbf{x}_{1}^{a}$, respectively, as shown in the right half of Fig. 1. Finally, $\mathbf{x}^{b}$ and $\mathbf{y}^{b}$ are obtained as

$$
\begin{aligned}
& \mathbf{x}^{b}=\left(\mathbf{s}_{\mathbf{x}} \oplus \mathbf{x}^{a} H_{a}^{T}\right)\left(H_{b}^{T}\right)^{-1} \\
& \mathbf{y}^{b}=\left(\mathbf{s}_{\mathbf{y}} \oplus \mathbf{y}^{a} H_{a}^{T}\right)\left(H_{b}^{T}\right)^{-1}
\end{aligned}
$$

Note that, although it is not shown explicitly in Fig. 1, likelihood calculation of the the all-zeros vector input to the decoder is done using the assumed cross-over probability $\varepsilon$ of the virtual BSC between sources $X$ and $Y$. Thus, the LLRs input to the decoder are $L=\log \frac{1-\varepsilon}{\varepsilon}$.

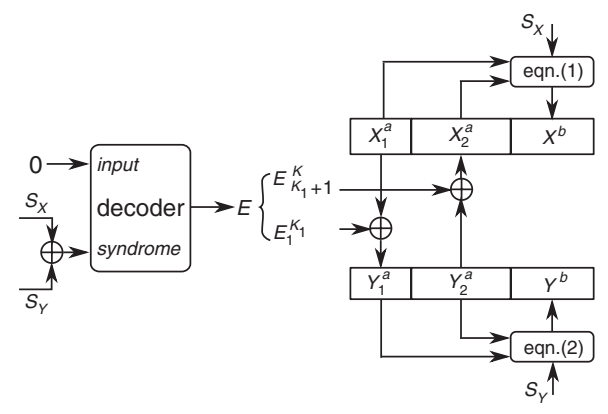

Fig. 1 Source decoding

A polar code is identified by a parameter set $\left(N, K, \mathcal{A}, \mathbf{u}_{\mathcal{A}^{c}}\right)$, where $N=2^{n}$ is the block length, $K$ is the code dimension, $\mathcal{A}$ is the information index set of size $K$ and $\mathbf{u}_{A^{c}}$ is the frozen bits vector of size $N-K$. The frozen bits $\mathbf{u}_{\mathcal{A}^{c}}$ identify a coset of the linear block code and can be used as a syndrome of the polar code [8]. An advantage of polar codes for this scheme is that the required syndrome decoding is readily available in the SC polar decoder. The SC decoder can decode as easily for a given $\mathbf{u}_{\mathcal{A}^{c}}$ as it can for the zero syndrome. However, this standard form of polar codes cannot be used in this method. Because, the second part of parity check matrix $\left(H_{b}\right)$ of a normal polar code is not invertible, thus the second part of decoding given by (1) cannot be performed. But, the systematic version of polar codes [12] can be used. In systematic polar coding, codeword $\mathbf{x}$ is split into two parts $\mathbf{x}=\left(\mathbf{x}_{\mathcal{B}}, \mathbf{x}_{\mathcal{B}^{c}}\right)$, where $\mathcal{B}$ is a $K$-bit subset of $\{1, \ldots, N\}$. Here, $\mathbf{x}_{\mathcal{B}}$ is the 'systematic' and $\mathbf{x}_{\mathcal{B}^{c}}$ is the 'parity' part. If $\mathcal{B}$ is selected suitably, the mapping $\mathbf{x}_{\mathcal{B}} \mapsto \mathbf{x}=\left(\mathbf{x}_{\mathcal{B}}, \mathbf{x}_{\mathcal{B}^{c}}\right)$ can be performed, for a fixed $u_{\mathcal{A}^{c}}$. This is the systematic polar encoding operation and can be done efficiently using a SC polar decoder [12]. The selection of the index set $\mathcal{B}$ is critical and it can be selected as the bit-reversed version of $\mathcal{A}$ [12].

Now, returning back to the non-asymmetric SW method of [11] described above, we set $\mathbf{x}^{a}=\mathbf{x}_{\mathcal{B}}, \mathbf{x}^{b}=\mathbf{x}_{\mathcal{B}^{c}}$ and $\mathbf{s}_{\mathbf{x}}=\mathbf{u}_{\mathcal{A}^{c}}$. This way we fulfil, the requirements of the method such that when $\mathbf{x}^{a}$ is decoded using the estimated error vector $\hat{\mathbf{e}}$, the rest, $\mathbf{x}^{b}$, can be recovered from $\mathbf{x}^{a}$ and $\mathbf{s}_{\mathbf{x}}$. Given $\mathbf{x}^{a}=\mathbf{x}_{\mathcal{B}}$ and $\mathbf{s}_{\mathbf{x}}=\mathbf{u}_{\mathcal{A}^{c}}$, computing $\mathbf{x}^{b}=\mathbf{x}_{\mathcal{B}^{c}}$ is nothing but a systematic polar encoding operation. We also use CRC to improve the short block length performance of the SCL decoder, which was originally proposed by the authors of [13] in a channel coding context. For the method proposed here, $L_{c r c}$-bit CRC of $N$-bit source block is calculated and transmitted in addition. With this modification, the $X$-encoder sends $\left(\mathbf{x}_{1}^{a}, \mathbf{s}_{\mathbf{x}}, \mathbf{c}_{\mathbf{x}}\right)$ and $Y$-encoder sends $\left(\mathbf{y}_{2}^{a}, \mathbf{s}_{\mathbf{y}}, \mathbf{c}_{\mathbf{y}}\right)$, where $\mathbf{c}_{\mathbf{x}}$ and $\mathbf{c}_{\mathbf{y}}$ are CRCs of $\mathbf{x}$ and $\mathbf{y}$, respectively. Since the CRC operation is linear, the CRC of error vector $\mathbf{e}=\mathbf{x} \oplus \mathbf{y}$ is $\mathbf{c}_{\mathbf{x}} \oplus \mathbf{c}_{\mathbf{y}}$. Thus, the SCL syndrome decoder can use this information when estimating the error vector. To match the required sum rate $R$, the channel code is adjusted so that $K=N(2-R)+2 L_{c r c}$.

Complexity of proposed method: Source encoding is essentially a syndrome calculation. It is done using a SC polar encoder which is of complexity $O(N \log N)$ [8]. Source decoding is done in two stages. First, the estimate of the error vector is calculated. This is the critical step of decoding where errors are introduced. Here we use a SC list decoder with a list size $L$. Hence, the complexity is $O(L N \log N)$ [13]. The second part of decoding involves calculation of $\mathbf{x}^{b}\left(\mathbf{f} \mathbf{y}^{b}\right)$ from $\mathbf{x}^{a}\left(\mathbf{y}^{a}\right)$ and $\mathbf{s}_{x}\left(\mathbf{s}_{y}\right)$ using (1) and (2). However, in practice matrix inversion and multiplication are not used. This calculation is effectively a 
systematic polar encoding operation and efficiently performed using a SC polar decoder. Thus, its complexity is $O(N \log N)$. Therefore, the total complexity of the source decoder is dominated by the first step which is of complexity $O(L N \log N)$.

Simulation results: The performance for $\left(R_{X}, R_{Y}\right)=(0.5,1)$, which corresponds to the asymmetric rate allocation, is presented in Fig. 2. Results for three different rate allocations are given in Table 1. A BER of $10^{-5}$ is considered to be lossless when determining the rate points. In all of the simulations, the sum rate of the code is kept at a defined constant value $(R=1.5)$ while $\varepsilon$ is varied to achieve different $H(X, Y)$ points. The BER values are averaged over $X$ and $Y$ sources. The SCL decoder of [13] with the addition of a 16-bit CRC (CCITT) is used. The list size is set to 32 . The code construction is done via the method proposed in [14] for a $\operatorname{BSC}(\varepsilon)$ and optimised to $\varepsilon=0.09$. From Table 1, it is observed that the performance of non-asymmetric rates are slightly inferior to the asymmetric case. This is expected, since in non-asymmetric cases estimation of the source $Y$ is also prone to errors, as opposed to the asymmetric case where no error is made for $Y$ Furthermore, these errors propagate to the recovery of $X$. Also note that the performance is the same for all non-asymmetric points.

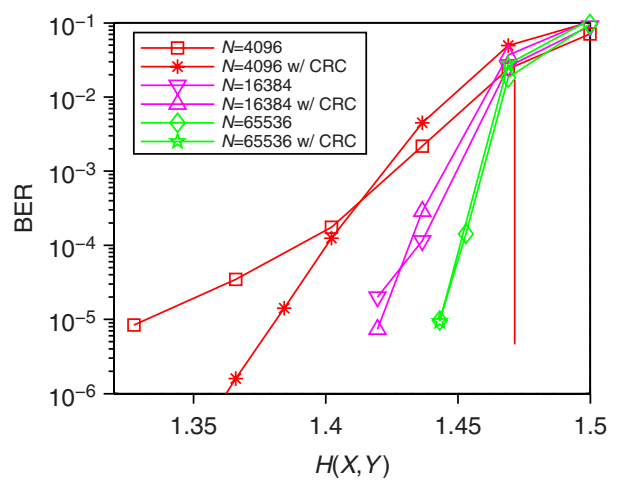

Fig. 2 BER performance of proposed method for $\left(R_{X}, R_{Y}\right)=(0.5,1)$

Table 1: Non-asymmetrical SW performance for $R=1.5(H(X, Y)$ values for a BER of $10^{-5}$ )

\begin{tabular}{|c|c|c|c|c|}
\hline$\left(\mathrm{R}_{\mathrm{X}}, \mathrm{R}_{\mathrm{Y}}\right) / \mathrm{N}$ & 2048 & 4096 & 16384 & 65536 \\
\hline$(0.500,1.000)$ & 1.351 & 1.381 & 1.421 & 1.443 \\
\hline$(0.625,0.875)$ & 1.336 & 1.365 & 1.405 & 1.435 \\
\hline$(0.750,0.750)$ & 1.335 & 1.364 & 1.405 & 1.435 \\
\hline
\end{tabular}

Conclusion: We have shown how polar codes can be used for constructing a non-asymmetric SW scheme for correlated sources with uniform marginals, using the general framework of [11]. A successive cancellation list decoder with the addition of CRC is used to achieve best performances. Although the performances are very good, they are slightly inferior to the best performances reported in the literature using turbo and LDPC codes [3, 6, 7]. However, there are some advantages in using polar codes. No modification is needed to the SC decoder since syndrome decoding is readily implemented. And the length of the syndrome can be modified easily and incrementally, which gives rise to flexible sum rate adaptation.

Acknowledgment: This work was supported by The Scientific and Technological Research Council of Turkey (TÜBITAK) under Project $110 \mathrm{E} 243$.

(C) The Institution of Engineering and Technology 2013

3 October 2012

doi: $10.1049 / \mathrm{el} .2012 .3495$

One or more of the Figures in this Letter are available in colour online.

S. Önay (Bilkent University, Ankara, Turkey)

E-mail: saygun@bilkent.edu.tr

\section{References}

1 Slepian, J.D., and Wolf, J.K.: 'Noiseless coding of correlated information sources', IEEE Trans. Inf. Theory, 1973, IT-19, pp. 471-480

2 Pradhan, S., and Ramchandran, K.: 'Distributed source coding using syndromes (DISCUS): design and construction'. Proc. of Data Compression Conf. (DCC'99), Snowbird, UT, USA, 1999, pp. 158-167

3 Liveris, A., Xiong, Z., and Georghiades, C.: 'Compression of binary sources with side information at the decoder using LDPC codes', IEEE Commun. Lett., 2002, 6, pp. 440-442

4 Varodayan, D., Aaron, A., and Girod, B.: 'Rate-adaptive distributed source coding using low-density parity-check codes'. Conf. Rec. of IEEE 39th Asilomar Conf. on Signals, Systems and Computers, Pacific Grove, CA, USA, 2005, November 2005, pp. 1203-1207

5 Roumy, A., Lajnef, K., and Guillemot, C.: 'Rate-adaptive turbosyndrome scheme for Slepian-Wolf coding'. Proc. of IEEE Asilomar Conf. on Signals, Systems, and Computers, Pacific Grove, CA, USA November 2007, pp. 545-549

6 Stankovic, V., Liveris, A., Xiong, Z., and Georghiades, C.: 'On code design for the Slepian-Wolf problem and lossless multiterminal networks', IEEE Trans. Inf. Theory, 2006, 52, pp. 1495-1507

7 Zamani, M., and Lahouti, F.: 'A flexible rate Slepian-Wolf code construction', IEEE Trans. Commun., 2009, 57, pp. 2301-2308

8 Arikan, E.: 'Channel polarization: a method for constructing capacity-achieving codes for symmetric binary-input memoryless channels', IEEE Trans. Inf. Theory, 2009, 55, pp. 3051-3073

9 Korada, S.B., and Urbanke, R.L.: 'Polar codes for Slepian-Wolf, Wyner-Ziv, and Gelfand-Pinsker'. IEEE Information Theory Workshop, (ITW), Cairo, Egypt, January 2010, pp. 1-5

10 Arikan, E.: 'Source polarization'. Proc. of IEEE Int. Symp. Information Theory, Austin, TX, USA, June 2010, pp. 899-903

11 Gehrig, N., and Dragotti, P.: 'Symmetric and asymmetric Slepian-Wolf codes with systematic and nonsystematic linear codes', IEEE Commun. Lett., 2005, 9, pp. 61-63

12 Arikan, E.: 'Systematic polar coding', IEEE Commun. Lett., 2011, 15, pp. $860-862$

13 Tal, I., and Vardy, A.: 'List decoding of polar codes'. Proc. 2011 IEEE Int. Symp. on Information Theory, (ISIT), St. Petersburg, Russia, August 2011, pp. 1-5

14 Tal, I., and Vardy, A.: 'How to construct polar codes', arXiv:1105.6164, May 2011 